\title{
BOUNDED FUNCTIONS WITH LARGE CIRCULAR VARIATION
}

\author{
GEORGE PIRANIAN ${ }^{1}$
}

In 1951, S. N. Mergeljan [1] proved that there exists a bounded holomorphic function $f$ for which

$$
\iint_{|z|<1}\left|f^{\prime}(z)\right| d S=\infty .
$$

An obvious geometric interpretation of $(1)$ is that the length $l(r)$ of the image of the circle $|z|=r$ grows so rapidly, as $r \rightarrow 1$, that $l(r)$ is not an integrable function of $r$.

An alternate geometric interpretation of (1) is that the length $V(f, \theta)$ of the image of the radius of $e^{i \theta}$ is not an integrable function of $\theta$. W. Rudin [2, Theorem III] has proved a proposition stronger than Mergeljan's, namely, that there exist Blaschke products $B(z)$ such that $V(B, \theta)=\infty$ for almost all $\theta$. It follows that there exists a function $f$, holomorphic in the unit disk $D$ and continuous in the closure of $D$, such that $V(f, \theta)=\infty$ for almost all $\theta$ [2, Theorem IV].

Both Mergeljan's and Rudin's arguments involve nonconstructive steps, and therefore they do not allow us to visualize the functions $f$ in terms of any of the customary representations. In this note, I give two explicit constructions that prove Mergeljan's result. Unfortunately, my examples are inadequate for Rudin's theorem.

We begin with the function $\left(a^{n}-z^{n}\right) /\left(1-a^{n} z^{n}\right)$, where $2^{-1 / n}<a<1$. We write $a^{n}=\alpha$ and $z^{n}=\zeta$, and we observe that for $0<\rho<\alpha$, the maximum and minimum values of $|(\alpha-\zeta) /(1-\alpha \zeta)|$ on the circle $|\zeta|=\rho$ are

$$
(\alpha+\rho) /(1+\alpha \rho) \text { and }(\alpha-\rho) /(1-\alpha \rho),
$$

respectively. The difference between the two moduli is $2 \rho\left(1-\alpha^{2}\right) /\left(1-\alpha^{2} \rho^{2}\right)$. Therefore the function $\left(a^{n}-z^{n}\right) /\left(1-a^{n} z^{n}\right)$, whose $2 n$ points of maximum and minimum modulus on the circle $|z|=r$ separate each other, maps that circle onto a curve of length greater than

$$
2 n \cdot 2 r^{n}\left(1-a^{2 n}\right) /\left(1-a^{2 n} r^{2 n}\right) \quad(0<r<a) .
$$

The integral of this quantity, taken over the interval $3^{-1 / n}<r<a$,

Received by the editors August 27, 1967.

1 This work was supported by the National Science Foundation. 
is greater than $K_{1} n(1-a)|\log n(1-a)|$, where $K_{1}$ is a constant independent of $a$ and $n$.

We now consider the Blaschke product

$$
B(z)=\prod \frac{a_{k}^{n_{k}}-z^{n_{k}}}{1-a_{k}^{n_{k}} z^{n_{k}}} .
$$

The product converges if $\sum n_{k}\left(1-a_{k}\right)<\infty$, in particular, if

$$
n_{k}\left(1-a_{k}\right)=1 / k(\log k)^{3 / 2} \quad(k=2,3, \cdots) .
$$

If moreover the sequence $\left\{n_{k}\right\}$ increases fast enough, we obtain disjoint intervals $r_{k}<r<a_{k}$ such that

$$
\begin{aligned}
\int_{r_{k}}^{a_{k}} \int_{0}^{2 \pi}\left|B^{\prime}\left(r e^{i \theta}\right)\right| r d \theta d r & >K_{2} n_{k}\left(1-a_{k}\right)\left|\log n_{k}\left(1-a_{k}\right)\right| \\
& >K_{2} / k(\log k)^{1 / 2}
\end{aligned}
$$

and Mergeljan's theorem is proved.

From our construction, we see immediately that there exists a continuous function $f$ satisfying condition (1). Indeed, it is sufficient to choose finite Blaschke products $B_{m}$ such that, for each of certain disjoint concentric annuli $A_{m}$,

$$
\iint_{A_{m}}\left|B_{m}^{\prime}\right| d S-\sum_{j \neq m} \iint_{A j}\left|B_{j}^{\prime}\right| d S>m^{3},
$$

and to take $f(z)=\sum m^{-2} B_{m}(z)$.

Our second example is based on the function

$$
g(z)=\exp \left(-a \frac{1+z^{n}}{1-z^{n}}\right) .
$$

Since the maximum and minimum modulus of $g(z)$ on the circle $\left|z^{n}\right|=\rho$ are

$$
\exp \left(-a \frac{1-\rho}{1+\rho}\right) \text { and } \exp \left(-a \frac{1+\rho}{1-\rho}\right)
$$

the function $g$ maps the circle $C_{r}$ onto a curve of length greater than

$$
2 n\left\{\exp \left(-a \frac{1-r^{n}}{1+r^{n}}\right)-\exp \left(-a \frac{1+r^{n}}{1-r^{n}}\right)\right\} .
$$

To estimate the integral of this lower bound over the interval $0<r<1$, 
we make the substitution $s=\left(1-r^{n}\right) /\left(1+r^{n}\right)$, and of the resulting integral

$$
4 \int_{0}^{1}\left(e^{-a s}-e^{-a / s}\right)(1-s)^{-1+1 / n}(1+s)^{-1-1 / n} d s
$$

we discard everything except the portion over $\left(0, a^{1 / 2}\right)$. We may then replace the algebraic factors by a constant, and the quantity to be determined is greater than

$$
K_{3} \int_{0}^{a^{1 / 2}}\left(e^{-a}-e^{-a / 8}\right) d s
$$

Consider separately each of the intervals $[(j-1) a, j a](j=1,2, \cdots$, $\left.\left[a^{-1 / 2}\right]\right)$. Since the minimum of the integrand in the $j$ th interval is

$$
e^{-a}-e^{-1 / j}>-a+j^{-1}-j^{-2},
$$

the value of the integral is greater than

$$
a \sum_{j=1}^{[a-1 / 2]}\left[-a+j^{-1}-j^{-2}\right]>K_{4} a|\log a| .
$$

Now, for $k=2,3, \cdots$, let $a_{k}=k^{-1}(\log k)^{-3 / 2}$, and let

$$
f(z)=\exp \left(-\sum a_{k} \frac{1+z^{n_{k}}}{1-z^{n_{k}}}\right) .
$$

If $n_{k} \rightarrow \infty$ fast enough, then $f$ again has the desired properties.

\section{REFERENCES}

1. S. N. Mergeljan, On an integral connected with analytic functions, Izv. Akad. Nauk SSSR Ser. Mat. 15(1951), 395-400. (Russian)

2. W. Rudin, The radial variation of analytic functions, Duke Math. J. 22(1955), 235-242.

UNIVERSITY OF MiChIGAN 